\title{
On the Extension of Invariant Partial States in Statistical Mechanics
}

\author{
G. G. EmCH*, H. J. F. KNops and E. J. Verboven \\ Instituut voor Theoretische Fysika \\ Universiteit Nijmegen, The Netherlands
}

Received August 22, 1967

\begin{abstract}
We consider a class of physical systems often encountered in such theories as statistical mechanics, namely those which admit an amenable group as a group of symmetries. We first establish the existence of invariant state(s) with respect to the symmetry group considered. We next introduce the notion of (extremal) potentially invariant states for an reduced description of the physical system considered; we then show that every such state can be extended to an (extremal) invariant state corresponding to a complete description.
\end{abstract}

\section{Introduction}

SEGaL's original proposition [1] to discuss in algebraic terms the structure of physical theories has been considered with great details in the recent years with the hope to bring at least some degree of order in the confusing puzzles offered to physicists by theories dealing with infinite systems, such as quantum field theory [2] or statistical mechanics [3].

Besides its formal achievements, the $C^{*}$-algebraic approach has been successfully tested in statistical mechanics on some models as simple as the ideal Bose- [4] and Fermi-gas [5]. Some success have also been registered when dealing with some very particular types of interacting systems, the most typical of which is the BCS-model [6]. These interacting models can be characterized in mathematical terms by saying that their equilibrium states are 'quasi-free states' [7]. In the every day language of the physicist this means that some kind of 'mean free field' approximation [9] (generalizing to other situations the Weiss' discussion of ferromagnets) becomes exact in the thermodynamical limit. However the 'mean free fields'-models are well known [10] to suffer from serious draw-backs in the general case. In particular, the behaviour of some of the thermodynamical functions is predicted quite wrongly by such theories. Consequently the next step, that the algebraic approach has to

* Permanent address: Department of Physics and Astronomy, the University of Rochester, Rochester, N. Y. 
make, is to escape from the frame of quasi-free states. In this respect, Robinson [7] made a significant, but none the less exasperating, discovery, namely that (for Boson-systems at least) there are only two classes of states in (equilibrium) statistical mechanics: those for which all truncated correlation functions of order $n>2$ identically vanish, and those for which there are always strictly non vanishing truncated functions of order greater than any positive number $N$.

The first class is that of the quasi-free states, the second class is the one we then would like to be able to deal with. In the face of the difficulty to deal with an infinite number of non-vanishing truncated functions one should ask what might be learned from the consideration of the first few non-vanishing truncated functions, and in which sense it is in principle possible to extend the information so gained to the unamputed states of the system considered.

A similar question might be asked in terms of the relation between states of macroscopic equilibrium and states of microscopic equilibrium. The present note gives a partial answer to this type of questions.

More specifically the type of questions considered here can be illustrated by the following example: Suppose that we are able, in one way or another, to construct the two-body correlation function of an infinite system. Suppose further that the correlation function so obtained is invariant with respect to space translations. One would like to know whether this correlation function can be considered as the restriction to two-particles observables of an homogeneous state of the whole system under consideration. From the general considerations presented in the next section we will see that not only the answer to this type of questions is positive, but moreover that if the correlation function is not only invariant, but 'extremal invariant' (in a sense which will be made precise) then it is possible to extend it to a state on the whole system, which satisfies again this same property.

\section{Properties of Invariant States}

Let us first express our physical problem in the proper mathematical language.

We suppose that the physical system under consideration is described

(i) by a $C^{*}$-algebra $\mathfrak{A}$ (for instance, the $C^{*}$-algebra of the quasi-local observables, or the $C^{*}$-algebra generated from the field operators); we can suppose without loss of generality that $\mathfrak{A}$ possesses an unit element, which we denote by $I$;

(ii) the convex set $\mathfrak{\Xi}$ of all states over $\mathfrak{A}$, i.e.

$$
\mathfrak{S}=\left\{\phi \in \mathfrak{A}^{*} \mid \phi(A) \geqq 0 A \in \mathfrak{A} \text { with } A \geqq 0 ; \phi(I)=1\right\} ;
$$


we further equip $\mathfrak{A}^{*}$ with the weak*-topology [11], i.e. the weakest topology relative to which the mappings $\phi \rightarrow \phi(A)$ are continuous for each $A$ in $\mathfrak{A}$ (this topology is also called [12] the $\mathfrak{A}$-topology on $\mathfrak{A}^{*}$ ); for what follows, it is important that $\subseteq$ is compact in this topology [13].

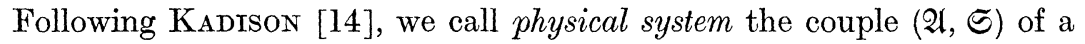
$C^{*}$-algebra $\mathfrak{A}$ and its set of states $\mathfrak{\mathcal { E }}$, the latter being equipped with the afore described topology. We find it more convenient for our purpose to work systematically in the 'Schrödinger picture'. Let us then make precise this latter statement; we actually just use the generalisation to an arbitrary topological group $G$, of the concept of 'dynamical system'

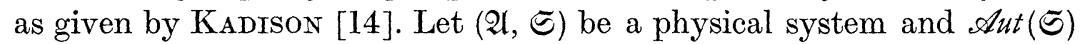
be the set of all $w^{*}$-continuous automorphisms of $\mathfrak{S}$, equipped with the topology naturally induced on it by the $w^{*}$-topology on $\widetilde{\Xi}$.

We now give for a symmetry group the following definition: we say that a physical system $(\mathfrak{A}, \mathfrak{S})$ admits the topological group $G$ as a symmetry group if we are given a homomorphism $v$ (in the sense of topological groups) from $G$ to $\mathscr{A u t}(\boldsymbol{\varsigma})$.

This means explicitely that (i) to each $g$ in $G$ we can attribute an affine $w^{*}$-unimorphism of $\mathfrak{\subseteq}$ on to itself, and (ii) for each $\phi$ in $\mathfrak{S}$ and each $A \in \mathfrak{A},\left(\boldsymbol{v}_{g} \phi\right)(A)$ is a continuous function on $G$. We denote this situation by

$$
\{\mathfrak{A}, \mathfrak{S}, v: G \rightarrow \mathscr{A u t}(\mathfrak{S})\} \text {. }
$$

We notice that the definition just given expresses all continuity conditions in terms of expectation values. It seems therefore hardly possible to weaken it anymore and still retain the essential features physically expected from a symmetry group. We now want to specialise the theory to the case where $G$ is an amenable group. To our knowledge this notion was used for the first time in physics by Doplcher, Kadison, Kastrer and Robinson [15], who also refer to the thesis of PIER [16]. Let us briefly recall what is meant by this notion which will play a central role in the sequel, as conjectured already for a particular case by Doplicher, KASTLER and RobINson [17].

Let $G$ be a locally compact group. We denote by $\mathscr{C}(G)$ the $C^{*}$-algebra, the elements of which are the continuous functions $\gamma$ from $G$ to $\mathbb{C}$ satisfying the condition:

$$
\|\gamma\| \equiv \operatorname{Sup}_{g \in G}|\gamma(g)|<\infty
$$

The addition, multiplication and involution in $\mathscr{C}(G)$ are defined as usual for complex valued functions. In particular $\mathscr{C}(G)$ possesses an identity $\delta$, namely the function which takes the value 1 for all $g$ in $G$.

To each $h$ in $G$ we associate two continuous $C^{*}$-automorphisms $h[]$ and []$h$ of $\mathscr{C}(G)$ defined respectively by:

$$
\begin{array}{ll}
(h[\gamma])(g)=\gamma(h g) & g \in G, \gamma \in \mathscr{C}(G) \\
([\gamma] h)(g)=\gamma(g h) & g \in G, \gamma \in \mathscr{C}(G) .
\end{array}
$$


We further consider the convex set $\Sigma(G)$ of all states $\eta$ on the $C *$-algebra $\mathscr{C}(G)$. The elements $\eta \in \Sigma(G)$ are also called 'mean over $G$ '.

To each $h$ in $G$ we associate two continuous *-unimorphisms $h$ [ ] and [ ] $h$ of $\Sigma(G)$ defined respectively by:

$$
\begin{array}{ll}
(h[\eta])(\gamma)=\eta(h[\gamma]) & \gamma \in \mathscr{C}(G), \eta \in \Sigma(G) \\
([\eta] h)(\gamma)=\eta([\gamma] h) & \gamma \in \mathscr{C}(G), \eta \in \Sigma(G) .
\end{array}
$$

A mean $\eta$ over $G$ is said to be left-invariant (resp. right-invariant) if $h[\eta]=\eta$ (resp. $[\eta] h=\eta)$ for all $h$ in $G$. It is said to be (two-sided) invariant if it is both left- and right-invariant. One can prove that if $G$ is a locally compact group the three following conditions are equivalent: (i) there exists at least one left-invariant mean over $G$, (ii) there exists at least one right-invariant mean over $G$, and (iii) there exists at least one invariant mean over $G$. A locally compact group which satisfies any one of the three above conditions is said to be amenable [16]. Let for instance $G$ be the additive group $\mathbb{R}$ of the real numbers (i.e. the 'translation group in one dimension'). $\mathscr{C}(\mathbb{R})$ is then the set of all complexvalued, bounded, continuous functions of one real variable. The 'ergodic mean' $\eta$ defined by :

$$
\eta(f)=\lim _{T \rightarrow \infty} \frac{1}{T} \int_{0}^{T} d t f(t)
$$

is an invariant mean over $\mathbb{R}$. We then conclude trivially that the translation group in one dimension is amenable. Similarly we have in fact that every locally compact abelian group is amenable, and that every compact group is amenable. Hence the following groups (which play an important role in statistical mechanics) are all amenable: the translation group in any number of dimensions, the gauge group, the rotation group in three dimensions, etc....

It is worth while noticing that the euclidean group in three dimensions is amenable, whereas neither the Galilei nor the Lorentz group are amenable.

Let now $\{\mathfrak{U}, \mathbb{S}, v: G \rightarrow \mathscr{A} u t(\mathfrak{S})\}$ be a physical system admitting an amenable group $G$ as a symmetry group. For any state $\phi$ in $\subseteq$ and any element $A$ in $\mathfrak{A}$ we consider the function $\left(\nu_{g} \phi\right)(A)$ from $G$ to $\mathbb{C}$. This function is continuous in $g$ by hypothesis. It is also bounded since

$$
\operatorname{Sup}_{g \in G}\left|\left(v_{g} \phi\right)(A)\right| \leqq \operatorname{Sup}_{g \in G}\left\|v_{g} \phi\right\| \cdot\|A\|=\|A\| \cdot
$$

Hence $\left(v_{g} \phi\right)(A)$ belongs to $\mathscr{C}(G)$. For any mean $\eta$ over $G$ let us form:

$$
\eta\left(\nu_{g} \phi(A)\right)
$$

and let us consider it as a mapping $\eta \circ \phi$ from $\mathfrak{A}$ to $\mathbb{C}$. From the linearity of $\nu_{g} \phi$ in $A$ and the linearity of $\eta$, we conclude that $\eta \circ \phi$ is a linear form 
on $\mathfrak{A}$. From the positivity of $\boldsymbol{\nu}_{g} \phi$ in $A$ and the positivity of $\eta$, we conclude that our form is positive. We further have $\left(v_{g} \phi\right)(I)=1$ for all $g$ in $G$. Hence $\left(v_{g} \phi\right)(I)$ is the unit element $\delta$ in $\mathscr{C}(G)$. Since $\eta$ is normalised to one, we have $\eta\left(\nu_{g} \phi(I)\right)=1$. Hence from the facts that $\eta$ is a state on $\mathscr{C}(G)$ and $\phi$ is a state on $\mathfrak{A}$, we concluded that $\eta \circ \phi$ is a state on $\mathfrak{A}$. One verifies easily that $v_{h}(\eta \circ \phi)=h[\eta] \circ \phi$ for all $h$ in $G$. Hence, if $\eta$ is a left-invariant mean over $G$ (such a mean always exists when $G$ is amenable), $\eta \circ \phi$ is an invariant state on $\mathfrak{A}$ whatever $\phi$ we chose in $\mathfrak{\Xi}$. We have then proved the following lemma:

Lemma 1. Let $\{\mathfrak{A}, \mathcal{E}, v: G \rightarrow \mathscr{A} u(\mathcal{E})\}$ be a physical system which admits the amenable group $G$ as a symmetry group. We have then: (i) if $\phi$ is a state in $\mathbb{S}$ and $\eta$ is a mean over $G$, then $\eta \circ \phi$ is a state in $\mathbb{G}$; (ii) if furthermore $\eta$ is left-invariant, then $\eta \circ \phi$ is invariant with respect to $G$; and in particular (iii) there always exists at least one state in $\mathfrak{\subseteq}$ which is invariant under $G$.

We notice that this lemma not only asserts the existence of invariant state(s) of a physical system which admits an amenable group $G$ as a symmetry group, but that it also gives a way to construct them. Starting from the Heisenberg picture, Doplicher [18] already established the existence of invariant states when $G$ is abelian, a fact which allowed him to use Markov-Kakutani fixed point theorem [12]. A similar existence theorem, valid for compact groups, was also indicated by RUELLE who uses an averaging method akin to ours. Our lemma indeed holds for all cases where an invariant mean exists on $G$, and it is then a natural extension of RuELre's and Doplicher's results. Incidentally we might mention that for the euclidean group in three-dimensions, the existence of invariant states could have been obtained by a combination of the results of these two authors, since $E^{3}$ is a semi-direct product of an abelian and a compact group. In closing this discussion of our lemma, we notice that the fact that we worked in the Schrödinger picture made our continuity assumptions some what weaker [14] and easier to justify from the physical point of view than those usually required when working in the Heisenberg picture.

Let now $(\mathfrak{A}, \mathfrak{S})$ be a physical system, $\mathfrak{M}$ be a self-adjoint, vectorial subspace of $\mathfrak{A}$, containing the unit element $I$ of $\mathfrak{A}$. For instance, $\mathfrak{M}$ could be the subspace generated by all monomials in the fields, up to degree $n$, or the subspace on which the $n$-body correlation functions are defined. It is hence physically relevant to concentrate our attention to the case where $\mathfrak{M}$ is just a subspace of $\mathfrak{A}$ and not necessarily a subalgebra of $\mathfrak{A}$. We define a partial state $f$ on $\mathfrak{M}$ as a linear form on $\mathfrak{M}$ which satisfies the following conditions:

(i) $f\left(M^{*}\right)=f(M)^{*} M \in \mathfrak{M}$. 
(ii) $f(M) \geqq 0 M \in \mathfrak{R} \cap \mathfrak{A}^{+}$where $\mathfrak{Q}^{+}$is the set of all positive elements of $\mathfrak{A}$.

(iii) $f(I)=1$.

We say that a state $\phi$ on $\mathfrak{Z}$ extends a partial state $f$ on $\mathfrak{M}$ whenever $\phi$, restricted to $\mathfrak{M}$, is equal to $f$. We have the following lemma:

Lemma 2. Let (2, ङ) be a physical system, $\mathfrak{2}$ be a self-adjoint vectorial subspace of $\mathfrak{A}$ containing the unit element. Then any partial state on $\mathfrak{M}$ can be extended to a state on $\mathfrak{A}$.

Proof. Dixmier [13] (2.10.1).

Let us now consider a physical system $\{\mathfrak{A}, \mathbb{E}, v: G \rightarrow \mathscr{A} u t(\mathfrak{S})\}$ which admits a topological group $G$ as a symmetry group. Let further $f$ be a partial state on a self-adjoint vectorial subspace $\mathfrak{W}$ of $\mathfrak{A}$ (with $I \in \mathfrak{W}$ ). From lemma 2 we know that there exists some extension(s) $\phi$ of $f$ to $\mathfrak{A}$. We say that $f$ is potentially-invariant with respect to $G$ if there exists among its extensions to $\mathfrak{A}$ at least one $\phi$ such that

$$
\left(v_{g} \phi\right)(M)=f(M) \quad M \in \mathfrak{R} .
$$

At this point it is perhaps worth to comment briefly on some physical aspects of this definition. As it is well-known from non-equilibrium statistical mechanics, there is in general not enough information in, say, the two-particles correlation function (at $t=0$ ) to allow, from the microscopic equation of the motion alone, the determination of the evolution in time of this correlation function: there exists indeed in general several states $\phi$ which extend a given partial state $f$ and these different states when propagated to $t>0$ correspond in general to different partial states. It is therefore out of question to define (in general) a strict notion of invariance of partial states, and the physical situation is best described in term of 'potentially invariant' partial states. There is however one particular case where the notion of potentially invariant partial states reduces to that of invariant partial state. Consider namely the case where $\mathfrak{M}$ is stable with respect to $G$. If then $f$ is potentially invariant on $\mathfrak{M}$, all its extensions $\psi$ to $\mathfrak{A}$ satisfy the condition $\left.\nu_{g} \psi\right|_{\mathfrak{M}}=f$ for all $g$, and then in this particular case one can state that $f$ is invariant under $G$ in the ordinary sense. This is however just a particular case and we want to be able to treat more general situations.

We further say that a partial state over $\mathfrak{M}$ is extremal-potentiallyinvariant if it cannot be written as a convex sum of potentially-invariant partial states on $\mathfrak{M}$.

We now want to prove the following theorem:

Theorem 1. Let $\{\mathfrak{A}, \mathfrak{S}, v: G \rightarrow \mathscr{A u t}(\mathfrak{S})\}$ be a physical system which admits an amenable group $G$ as a symmetry group. Let $\mathfrak{M}$ be a self-adjoint, vectorial subspace of $\mathfrak{A}$, containing the unit element of $\mathfrak{A}$. Then (i) any potentially-invariant partial state $f$ on $\mathfrak{R}$ can be extended to an invariant 
state $\phi$ on $\mathfrak{A}$, and (ii) if $f$ is moreover extremal-potentially-invariant, then $\phi$ can always be chosen to be extremal invariant.

Proof. Let $f$ be a potentially invariant partial state on $\mathfrak{M}$. From the definition of $f$ we know that there exists at least one state $\phi$ on $\mathfrak{A}$ such that $v_{g} \phi$, when restricted to $\mathfrak{M}$, is equal to $f$ for all $g$ in $G$. For each $M$ in $\mathfrak{M}$ consider the function $\left(v_{g} \phi\right)(M)=f(M)$. This is a multiple of the unit element $\delta$ in $\mathscr{C}(G)$. Hence the state $\eta \circ \phi$ when restricted to $\mathfrak{M}$ is still equal to $f$, whatever mean $\eta$ over $G$ we chose. If $\eta$ is furthermore leftinvariant (such a invariant mean exists since $G$ is amenable) $\eta \circ \phi$ is an invariant state on $\mathfrak{A}$ which extends $f$. This proves the first part of our theorem. Let us now suppose that $f$ is an extremal potentially invariant partial state on $\mathfrak{M}$. Let us denote by $\{G\}$ the set of all states on $\mathfrak{A}$ which are invariant with respect to $G$, and by $\{f\}$ the set of all states on $\mathfrak{A}$ which extend $f .\{G\} \cap\{f\}$ is then the set of all $G$-invariant states on $\mathfrak{A}$ which extend $f$. It is non-void as we just proved. It is moreover convex, closed in the $w^{*}$-topology and bounded in the metric topology. It is therefore [12] a convex, $w^{*}$-compact subset of the linear space $\mathfrak{A}^{*}$ which is itself locally convex for the $w^{*}$-topology [12]. From Krein-Milman theorem [12] we know then that $\{G\} \cap\{f\}$ contains its extremal points. Let $\phi$ be any of them. We want to show that $\phi$ is also an extremal point of $\{G\}$. Suppose the contrary and write $\phi=\alpha \phi_{1}+(1-\alpha) \phi_{2}$ with $0<\alpha<1$ and $\phi_{1}, \phi_{2} \in\{G\}$. By restricting this equality to $\mathfrak{M}$ we contradict the assumption that $f$ is extremal potentially invariant unless both $\phi_{1}$, and $\phi_{2}$, when restricted to $\mathfrak{M}$, are equal to $f$. Therefore both $\phi_{1}$, and $\phi_{2}$ have to belong to $\{f\}$ and therefore to $\{G\} \cap\{f\}$ which contradicts our choice of $\phi$ as an extremal invariant point of $\{G\} \cap\{f\}$ unless $\phi_{1}=\phi_{2}=\phi$. Consequently $\phi$ is an extremal point of $\{G\}$. This achieves the proof of our theorem.

We notice that this theorem is a generalisation of a theorem mentionned by Doplicher, Kastuer and Robinson [17]. Our generalisation essentially consists in the fact we neither require that $\mathfrak{M}$ is a subalgebra of $\mathfrak{A}$ (so that we can consider states given by $n$-particles correlation functions or $n$-points Wightman functions) or that $\mathfrak{M}$ is stable with respect to $G$ (so that we can consider 'ergodic states' for the time-evolution of interacting systems).

We might also point out that the well-known Markov-Kakutani fixed point theorem [12] can be used to produce an alternative proof of the first part of our theorem in the particular case where $G$ is abelian. It is perhaps worth mentioning that this proof, in opposition to that given above, does not need any assumption on the topological structure of $G$. Let us then briefly sketch this alternative proof. Let $f$ be any potentially invariant partial state on $\mathfrak{R}$ and $\{f\}^{\prime}$ be the set of all states $\phi$ in $\mathfrak{S}$ which extend $f$ and satisfy to $\left.\nu_{g} \phi\right|_{\mathfrak{m}}=f$ for all $g$ in $G$. $\{f\}^{\prime}$ is non- 
void (since $f$ is potentially invariant), convex, closed in the $w^{*}$-topology and bounded in the metric topology. It is then a $w^{*}$-compact, convex subset of the linear space $\mathfrak{A}^{*}$ which we now consider as equipped with the $w^{*}$-topology. $\{f\}^{\prime}$ is moreover stable with respect to $G$. We can therefore use Markov-Kakutani [12] theorem to conclude the existence of at least one invariant state $\phi$ on $\mathfrak{A}$, extending $f$. In the even more particular case where we have further that $\mathfrak{M}$ is stable with respect to $G$, one has in the above proof $\{f\}^{\prime}=\{f\}$.

\section{Conclusion}

The mathematical results of this note are expressed in the lemma 1 and the theorem 1. The physical interpretations we give of these results is as follows. Lemma 1 establishes the existence of invariant state(s) for a class of groups wide enough for the purpose of statistical mechanics, namely the class $\{G\}$ of amenable groups. The connection between ergodic theory (considered as an averaging procedure) and invariant states is indicated. Theorem 1 shows that all (extremal) potentially invariant partial states on certain subspaces $\mathfrak{M}$ of a $C^{*}$-algebra $\mathfrak{A}$ can be obtained as restrictions to $\mathfrak{W}$ of (extremal) invariant states on the whole of $\mathfrak{A}$. The theory is formulated in such a way as to cover the following particular cases, which might be of interest to statistical mechanics. Firstly, the algebra $\mathfrak{A}$ can be taken as the algebra of quasi-local observables, or the algebra of the quasi-local fields (either bosons or fermions). Secondly, $G$ can be chosen to be the translation group in time, the gauge group, the translation group in three-dimensions, the rotation group in three dimensions, the euclidean group in three-dimensions, etc. . . Thirdly, $\mathfrak{M}$ can be realised as the subspace generated by the monomials up to order $n$ in the one-particle observables or in the field operators. As a particular case, $\mathfrak{M}$ can also be a subalgebra of $\mathfrak{A}$, e.g. $\mathfrak{M}$ could be the set of all macroscopic intensive observables.

Acknowledgements. At an early stage of the research discussed in this note, two of us (G. G. E. and E. J. V.) had a very stimulating discussion with David RUELLE. In the course of this discussion, held at the Second NUFFIC Summer School (1967), a proof of the theorem was outlined for the particular case mentionned at the end of section 2 .

\section{Bibliography}

1. Segal I. E.: Ann Math. 48, 930 (1947).

2. See for instance: HAAG, R., and D. Kastler: J. Math. Phys. 3, 248 (1964).

3. See for instance: HaAg, R., N. M. Hugenholtz, and M. Winnink: Commun. Math. Phys. 5, 215 (1967), and ref. quoted there in.

4. Araki, H., and E. J. Woods: J. Math. Phys. 4, 637 (1963); (see also Verbedre, A. F., and E. J. Verboven: to appear in Physica).

5. - , and W. Wyss: Helv. Phys. Acta 37, 136 (1964). 
6. HAaG, R.: Nuovo Cimento 25, 287 (1962); Ezawa, H.: J. Math. Phys. 5, 1078 (1964); Emch, G., and M. Guenin: J. Math. Phys. 7, 916 (1966); Thirring, W., and A. Wehrl: Commun. Math. Phys. 4, 303 (1967); Balslev, E., and A. Verbeure: IHES' preprint 1967.

7. Robinson, D. W.: Commun. Math. Phys. 1, 89 (1965).

8. - Commun. Math. Phys. 1, 159 (1965).

9. Емсн, G. G.: J. Math. Phys. 8, 13 and 19 (1967).

10. See for instance: Brout, R.: Phase transitions. New York: W. A. Benjamin 1965.

11. Hille, E., and R. S. Phillies: Functional analysis and semi-groups, Am. Math. Soc. Coll. Publ. Vol. XXXI, Providence, R. I. 1957. In particular, section II. 2. 10.

12. Dunford, N., and J. T. Schwartz: Linear operators, New York: Interscience Publ. Inc. 1964. In particular, Chap. V.

13. See for instance: Drxmiter, J.: Les $C^{*}$-algèbres et leurs représentations. Paris: Gauthier-Villars 1964.

14. Kadison, R. V.: Topology 3, Suppl. 2, 177 (1965).

15. Doplicher, S., R. V. Kadison, D. Kastler, and D. W. Robinson: preprint 1966.

16. Pier, J. P.: Thèse de 3ème cycle, Nancy, 1965.

17. Doplicher, S., D. Kastler, and D. W. Robinson: Commun. Math. Phys. 3, 1 (1966). (see in particular Th. 8 and their 'note added in proof').

18. - Commun. Math. Phys. 1, 1 (1966).

19. Ruelle, D.: Commun. Math. Phys. 3, 133 (1966). 